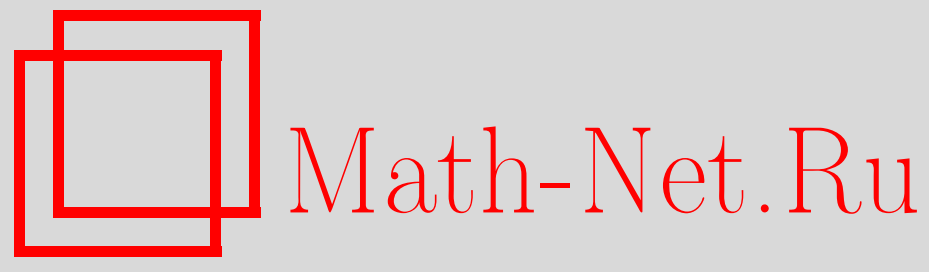

Н. Ж. Наурызбаев, Н. Темиргалиев, О порядке дискрепанса сетки Смоляка, Матем. заметки, 2009, том 85, выпуск 6, 947-950

DOI: https://doi.org/10.4213/mzm7661

Использование Общероссийского математического портала Math-Net.Ru подразумевает, что вы прочитали и согласны с пользовательским соглашением http://www . mathnet.ru/rus/agreement

Параметры загрузки:

IP : 18.207 .199 .55

26 апреля 2023 г., 17:35:10

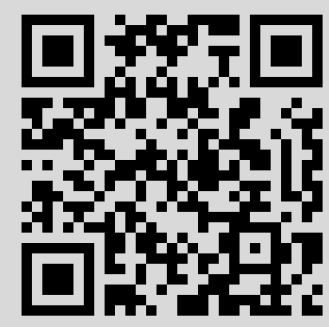




\section{О порядке дискрепанса сетки Смоляка}

\section{Н. Ж. Наурызбаев, Н. Темиргалиев}

Квадратурной формулой Смоляка (см. [1]-[4]) 1-периодической по каждой из $s$ переменных функции $f\left(x_{1}, \ldots, x_{s}\right)$ называют сумму $(s=2,3, \ldots, q=s, s+1, \ldots)$

$$
\Lambda_{q}(f)=\sum_{\ell=0}^{s-1}(-1)^{\ell}\left(\begin{array}{c}
s-1 \\
\ell
\end{array}\right) \sum_{\substack{\nu_{j} \in \mathbb{Z}: \nu_{j} \geqslant 0, j=1, \ldots, s, \nu_{1}+\cdots+\nu_{s}=q-\ell}} \frac{1}{2^{q-\ell}} \sum_{k_{1}=1}^{2^{\nu_{1}}} \cdots \sum_{k_{s}=1}^{2^{\nu_{s}}} f\left(\frac{k_{1}}{2^{\nu_{1}}}, \ldots, \frac{k_{s}}{2^{\nu_{s}}}\right) .
$$

Система узлов (без повторений, в силу 1-периодичности компоненты узлов 0 и 1 отождествлены, из них в качестве компонент оставлено только 1) из (1) образует множество

$$
\begin{aligned}
\left\{t_{k}\right\}_{k=1}^{N} \equiv\left\{\left(\frac{2 \mu_{1}-1}{2^{\nu_{1}}}, \frac{2 \mu_{2}-1}{2^{\nu_{2}}}, \ldots, \frac{2 \mu_{s-1}-1}{2^{\nu_{s-1}}}, \frac{\mu_{s}}{2^{\nu_{s}}}\right):\right. \\
\left.1 \leqslant \mu_{j} \leqslant \max \left\{1,2^{\nu_{j}-1}\right\}, \nu_{j} \geqslant 0, j=1,2, \ldots, s-1,1 \leqslant \mu_{s} \leqslant 2^{\nu_{s}}, \sum_{j=1}^{s} \nu_{j}=q\right\}
\end{aligned}
$$

мощности $N=N_{s}(q) \underset{s}{\asymp} 2^{q} q^{s-1}$. Здесь и всюду ниже через $c(\ldots)$ будем обозначать некоторые положительные величины, разные, вообще говоря, в различных формулах и зависящие лишь от указанных в скобках параметров. При положительном $A$ и любом $B$ запись $B \underset{\alpha, \beta, \ldots}{\ll} A$ будет означать $|B| \leqslant c(\alpha, \beta, \ldots) A$. При положительных $A$ и $B$ запись $A \underset{\alpha, \widehat{\beta}, \ldots}{\asymp} B$ означает $A \underset{\alpha, \beta, \ldots}{\ll} B \underset{\alpha, \beta, \ldots}{\ll} A$.

Заметим, что в силу (2) квадратурная формула (1) записывается в виде суммы без повторяющихся узлов

$$
\begin{aligned}
& \Lambda_{q}(f)=\sum_{\substack{\left(\nu_{1}, \ldots, \nu_{s}\right) \in \mathbb{Z}_{0}^{s}: \\
\nu_{1}+\cdots+\nu_{s} \leqslant q}} \sum_{\substack{\left(\mu_{1}, \ldots, \mu_{s}\right) \in \mathbb{Z}_{+}^{s}: \\
1 \leqslant \mu_{j} \leqslant \max \left\{1,2^{\nu_{j}-1}\right\}, j=1, \ldots, s-1,}} \sum_{\ell=0}^{s-1} \frac{(-1)^{\ell}}{2^{q-\ell} \ell !} \\
& 1 \leqslant \mu_{s} \leqslant \max \left\{1,2^{q-\left(\nu_{1}+\cdots+\nu_{s-1}+\nu_{s}\right)-1}\right\} \\
& \times\left(\frac{\left(s-1+\nu_{s}-\ell\right) !}{(s-1-\ell) !\left(\nu_{s}-\ell\right) !}\right)^{*} f\left(\frac{2 \mu_{1}-1}{2^{\nu_{1}}}, \ldots, \frac{2 \mu_{s-1}-1}{2^{\nu_{s-1}}}, \frac{2 \mu_{s}-1}{2^{q-\left(\nu_{1}+\cdots+\nu_{s-1}+\nu_{s}\right)}}\right),
\end{aligned}
$$

где $\mathbb{Z}_{0}^{s}$ и $\mathbb{Z}_{+}^{s}$ состоят из всех точек целочисленной $s$-мерной решетки $\mathbb{Z}^{s}$ с неотрицательными и положительными компонентами соответственно, а

$$
\left(\frac{\left(s-1+\nu_{s}-\ell\right) !}{(s-1-\ell) !\left(\nu_{s}-\ell\right) !}\right)^{*}= \begin{cases}\frac{\left(s-1+\nu_{s}-\ell\right) !}{(s-1-\ell) !\left(\nu_{s}-\ell\right) !}, & \text { если } \nu_{s} \geqslant \ell, \\ 0, & \text { если } \nu_{s}<\ell .\end{cases}
$$

В данной работе исследуется задача о степени равномерности распределения сетки Смоляка в единичном кубе.

Количественным показателем свойства равномерности распределения сетки $\xi_{1}, \ldots, \xi_{N}$ на единичном кубе $[0,1]^{s}$ является ее дискрепанс (наибольшее отклонение доли точек сетки, попадающих в произвольный параллелепипед со сторонами, параллельными сторонам единичного куба, от доли объема этого параллелепипеда по отношению к объему 
единичного куба)

$$
\begin{gathered}
D_{s}\left(\xi_{1}, \ldots, \xi_{N}\right)=\sup \left\{\left|\frac{A_{J}}{N}-\frac{|J|}{1}\right|=\left|\frac{1}{N} \sum_{k=1}^{N} \chi_{J}\left(\xi_{k}\right)-\int_{[0,1]^{s}} \chi_{J}(x) d x\right|:\right. \\
\left.J=\left(a_{1}, b_{1}\right] \times \cdots \times\left(a_{s}, b_{s}\right] \subset(0,1]^{s}\right\},
\end{gathered}
$$

где $\chi_{\Omega}(x)$ - характеристическая функция множества $\Omega, A_{J}$ - количество точек $\xi_{1}, \ldots, \xi_{N}$, содержащихся в $J,|J|$ - объем $J$.

Из результатов Рота [5] следует, что для всяких $s, s=1,2, \ldots$, и $N, N=1,2, \ldots$, всякой конечной последовательности $\xi_{1}, \ldots, \xi_{N}$ из $[0,1]^{s}$ и некоторого положительного $c(s)$ выполняется неравенство

$$
D_{s}\left(\xi_{1}, \ldots, \xi_{N}\right) \geqslant c(s) \frac{(\ln N)^{(s-1) / 2}}{N} .
$$

Вместе с тем, существует последовательность сеток $\left\{\xi_{k}\right\}_{k=1}^{N}$ с достаточно плотным количеством узлов $N$ такая, что для дискрепанса $D_{s}$ выполняется неравенство

$$
D_{s}\left(\xi_{1}, \ldots, \xi_{N}\right) \leqslant \widetilde{c}(s) \frac{\ln ^{\beta} N}{N} .
$$

Такие сетки называют равномерно распределеннъми.

Известно, что квадратурная формула (1) обладает хорошими аппроксимативными свойствами, близкими к оптимальным (см., например, [1]-[4] и имеющуюся в них библиографию). Вместе с тем, как оказалось, сетка Смоляка на единичном кубе распределена очень плохо. Именно, справедлива

Теорема. Для сетки Смоляка $B_{N}=\left\{t_{k}\right\}_{k=1}^{N}$ из (2) имеет место двусторонняя оценка

$$
D_{s}\left(\left\{t_{k}\right\}_{k=1}^{N}\right) \underset{s}{\asymp} \log ^{-1} N, \quad q \geqslant s, \quad N=N_{s}(q) \underset{s}{\asymp} 2^{q} q^{s-1} .
$$

В связи с соотношением (4) заметим, что по определению (3) дискрепанс $D_{s}\left(\left\{t_{k}\right\}_{k=1}^{N}\right)$ не может иметь хорошие свойства, поскольку к интегралу $\int_{[0,1]^{s}} \chi_{J}(x) d x$ применяется не квадратурная формула Смоляка (1), оптимальная в степенной шкале, а иная - с той же сеткой, но с другими, а именно, равными весами. В данной теореме, по существу, выясняется величина потери от замены весов.

Наметим основные моменты доказательства сформулированной теоремы (краткий анализ развития методов оценок дискрепансов и перспектив темы дан в [6]).

СХема ДОКАЗАТЕЛЬСТвА теОРемы. Ниже через $|B|$ будем обозначать количество точек конечного множества $B$, через $[x]$ и $\{x\}$ - целую и дробную части действительного числа $x$.

Оценка сверху. Для любой конечной последовательности $\xi_{1}, \ldots, \xi_{N}$ из $(0,1]^{s}$ верны неравенства (см. [7; с. 101-104])

$$
D_{s}^{*}\left(\xi_{1}, \ldots, \xi_{N}\right) \leqslant D_{s}\left(\xi_{1}, \ldots, \xi_{N}\right) \leqslant 2^{s} D_{s}^{*}\left(\xi_{1}, \ldots, \xi_{N}\right),
$$

где

$$
D_{s}^{*}\left(\xi_{1}, \ldots, \xi_{N}\right)=\sup _{\substack{J=\left(\alpha_{1}, 1\right] \times \ldots \times\left(\alpha_{s}, 1\right] \subset(0,1]^{s} \\ 0 \leqslant \alpha_{1}<1, \ldots, 0 \leqslant \alpha_{s}<1}}\left|\frac{1}{N} \sum_{i=1}^{N} \chi_{J}\left(\xi_{i}\right)-\left(1-\alpha_{1}\right) \cdots\left(1-\alpha_{s}\right)\right| .
$$

Пусть $s \geqslant 2$. Пусть даны числа $\alpha_{j}, 0 \leqslant \alpha_{j}<1, j=1, \ldots, s$, целое число $q \geqslant s$. Положим

$$
I_{s} \equiv I_{s}(\alpha)=I_{s}\left(\alpha_{1}, \alpha_{2}, \ldots, \alpha_{s}\right)=\left(\alpha_{1}, 1\right] \times\left(\alpha_{2}, 1\right] \times \cdots \times\left(\alpha_{s}, 1\right],
$$


тогда

$$
\begin{aligned}
&\left|B_{N} \cap I_{s}(\alpha)\right|=\sum_{k=1}^{N} \chi_{I_{s}(\alpha)}\left(t_{k}\right) \\
&=\sum_{\lambda_{1}=0}^{q} \cdots \sum_{\lambda_{s-1}=0}^{q} \prod_{j=1}^{s-1}\left(\left[2^{q-\lambda_{j}-1}+\frac{1}{2}\right]-\left[\alpha_{j} 2^{q-\lambda_{j}-1}+\frac{1}{2}\right]\right) \\
& \quad \times\left[2^{q-\sum_{j=1}^{s-1}\left(q-\lambda_{j}\right)}-\left[\alpha_{s} 2^{q-\sum_{j=1}^{s-1}\left(q-\lambda_{j}\right)}\right]\right] .
\end{aligned}
$$

Отсюда, при $\alpha_{1}=\cdots=\alpha_{s}=0$ получим точное значение количества точек $N=N_{s}(q)$ сетки $B_{N}$ :

$$
N=N_{s}(q)=\sum_{\lambda_{1}=0}^{q} \cdots \sum_{\lambda_{s-1}=0}^{q} \prod_{j=1}^{s-1}\left[2^{q-\lambda_{j}-1}+\frac{1}{2}\right]\left[2^{q-\sum_{l=1}^{s-1}\left(q-\lambda_{l}\right)}\right] \underset{s}{\asymp} 2^{q} q^{s-1} .
$$

Положим

$$
g_{s}(\alpha)=g\left(\alpha_{1}, \ldots, \alpha_{s}\right)=\left(1-\alpha_{1}\right) \cdots\left(1-\alpha_{s}\right)-\frac{1}{N} \sum_{k=1}^{N} \chi_{I_{s}(\alpha)}\left(t_{k}\right)
$$

и определим функцию

$$
\begin{aligned}
\Phi_{\alpha}\left(\lambda_{1}, \ldots, \lambda_{s-1}\right)=(1 & \left.-\alpha_{1}\right) \cdots\left(1-\alpha_{s}\right) \prod_{j=1}^{s-1}\left[2^{q-\lambda_{j}-1}+\frac{1}{2}\right]\left[2^{q-\sum_{k=1}^{s-1}\left(q-\lambda_{k}\right)}\right] \\
& -\prod_{j=1}^{s-1}\left(\left[2^{q-\lambda_{j}-1}+\frac{1}{2}\right]-\left[\alpha_{j} 2^{q-\lambda_{j}-1}+\frac{1}{2}\right]\right) \\
& \times\left[2^{q-\sum_{k=1}^{s-1}\left(q-\lambda_{k}\right)}-\left[\alpha_{s} 2^{q-\sum_{k=1}^{s-1}\left(q-\lambda_{k}\right)}\right]\right] .
\end{aligned}
$$

Тогда

$$
N g_{s}(\alpha)=\sum_{\lambda_{j}=0}^{q} \cdots \sum_{\lambda_{s-1}=0}^{q} \Phi_{\alpha}\left(\lambda_{1}, \ldots, \lambda_{s-1}\right)
$$

Сначала определяется сумма $A_{0}(\alpha, q)$ :

$$
A_{0}(\alpha, q)=N g_{s}(\alpha)-\sum_{\lambda_{1}=1}^{q} \ldots \sum_{\lambda_{s-1}=1}^{q} \Phi_{\alpha}\left(\lambda_{1}, \ldots, \lambda_{s-1}\right)
$$

как сумма всех таких $\Phi_{\alpha}\left(\lambda_{1}, \ldots, \lambda_{s-1}\right)$, что по крайней мере одно из $\lambda_{j}$ обращается в нуль, и доказывается, что

$$
\left|A_{0}(\alpha, q)\right| \underset{s}{\ll} 2^{q} .
$$

Далее, определяется $A_{1}(\alpha, q)$ :

$$
\begin{aligned}
& A_{1}(\alpha, q)=\left(N g_{s}(\alpha)-A_{0}(\alpha, q)\right) \\
&-\sum_{\lambda_{1}=1}^{q-1} \ldots \sum_{\lambda_{s-1}=1}^{q-1}\left\langle\left(1-\alpha_{1}\right) \cdots\left(1-\alpha_{s}\right) \prod_{j=1}^{s-1}\left[2^{q-\lambda_{j}-1}+\frac{1}{2}\right]\left[2^{q-\sum_{k=1}^{s-1}\left(q-\lambda_{k}\right)}\right]\right. \\
&-\prod_{j=1}^{s-1}\left(2^{q-\lambda_{j}-1}-\left[\alpha_{j} 2^{q-\lambda_{j}-1}+\frac{1}{2}\right]\right) \\
&\left.\times\left[2^{q-\sum_{k=1}^{s-1}\left(q-\lambda_{k}\right)}-\left[\alpha_{s} 2^{q-\sum_{k=1}^{s-1}\left(q-\lambda_{k}\right)}\right]\right]\right\rangle
\end{aligned}
$$


как сумма, составленная из всех таких слагаемых $\Phi_{\alpha}\left(\lambda_{1}, \ldots, \lambda_{s-1}\right)$, что по крайней мере одно $\lambda_{j}$ равно $q$, и устанавливается, что

$$
\left|A_{1}(\alpha, q)\right| \leqslant \sum_{l=1}^{s-1}\left(\begin{array}{c}
s-1 \\
l
\end{array}\right) N_{s-1}(q)=\left(2^{s-1}-1\right) N_{s-1}(q) .
$$

И, наконец, для $A_{2}(\alpha, q)=N g_{s}(\alpha)-A_{0}(\alpha, q)-A_{1}(\alpha, q)$ имеем

$$
\left|A_{2}(\alpha, q)\right| \underset{s}{\ll} 2^{q} q^{s-2} \underset{s}{\asymp} N_{s-1}(q) .
$$

В итоге, в силу (6), (7) и (8) приходим к соотношениям

$$
\begin{aligned}
D_{s}\left(\left\{t_{k}\right\}_{k=1}^{N}\right) & \leqslant 2^{s} \sup _{\alpha \in[0,1)^{s}}\left|g_{s}(\alpha)\right| \leqslant 2^{s} \sup _{\alpha \in[0,1)^{s}} \frac{\left|A_{0}(\alpha, q)\right|+\left|A_{1}(\alpha, q)\right|+\left|A_{2}(\alpha, q)\right|}{N_{s}(q)} \\
& \ll \frac{N_{s-1}(q)}{N_{s}(q)} \asymp \frac{2^{q} q^{s-2}}{2^{q} q^{s-1}}=\frac{1}{q} \asymp \frac{1}{s} .
\end{aligned}
$$

Этим завершим схему доказательства теоремы в части получения оценки сверху.

Оценка снизу. Имеем

$$
\sup _{\alpha \in[0,1)^{s}}\left|g_{s}(\alpha)\right| \geqslant \sup _{\alpha_{1} \in\left[\left(2^{q}-1\right) / 2^{q}, 1\right)}\left|g_{s}\left(\alpha_{1}, 0, \ldots, 0\right)\right| \geqslant\left|\frac{N_{s-1}(q)}{N_{s}(q)}-\left(1-\alpha_{1}\right) \cdot 1 \cdots 1\right|,
$$

откуда в силу произвольности $\alpha_{1}$ и из (5) получаем

$$
D_{s}\left(\left\{t_{k}\right\}_{k=1}^{N}\right) \geqslant \frac{N_{s-1}(q)}{N_{s}(q)} \underset{s}{\varsigma} \frac{2^{q} q^{s-2}}{2^{q} q^{s-1}}=\frac{1}{q} \asymp \frac{1}{s} \frac{1}{\ln N} .
$$

Оценка снизу, тем самым и теорема, с точностью до промежуточных утверждений, доказана.

В заключение заметим, что результат данного сообщения был анонсирован в [8], где показатель $-(s-1)$ в оценке дискрепанса надлежит заменить на -1 (то же относится и к [9], где в наших обозначениях утверждается, что для сетки Смоляка выполнено неравенство $\left.D_{s}\left(\left\{t_{k}\right\}_{k=1}^{N}\right) \ll(\ln N)^{-(s-1)}\right)$.

Авторы благодарят рецензента за полезные замечания.

\section{СПИСОК ЦИТИРОВАННОЙ ЛИТЕРАТУРЫ}

[1] С. А. Смоляк, Докл. АН СССР, 148:5 (1963), 1042-1045. [2] G. W. Wasilkowski, H. Wozniakowski, J. Complexity, 11:1 (1995), 1-56. [3] Н. Темиргалиев, Докл. PAH, 393:5 (2003), 605-608. [4] Н. Темиргалиев, Вестн. Евразийского наи. ун-та, 2007, № 2, 19-51. [5] K. F. Roth, Mathematika, 1:2 (1954), 73-79. [6] К.Ф. Рот, Математика. Границы и перспективы, ФАЗИС, М., 2005, 375-394. [7] Л. Кейперс, Г. Нидеррейтер, Равномерное распределение последовательностей, Наука, М., 1985. [8] Н. Ж. Наурызбаев, Н. Темиргалиев, Тезисы Международной научной конференции "Современные проблемы математики, механики, информатики", ТулГУ, Тула, 2005, 125-127. [9] Н. М. Добровольский, А. Р. Есаян, Р. Р. Яфаева, Тезисы докладов Всеросийской научной конференции "Современные проблемы математики, механики, информатики", ТулГУ, Тула, 2002, 18-20.

\section{Н. Ж. Наурызбаев}

Поступило

Евразийский национальный университет, г. Астана

09.11 .2008

E-mail: nngmath@mail.ru

\section{Н. Темиргалиев}

Евразийский национальный университет, г. Астана

E-mail: ntmath@mail.ru 